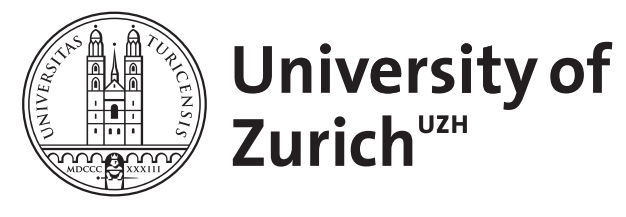

\title{
Effect of low mass dark matter particles on the Sun
}

\author{
Taoso, M ; Iocco, F ; Meynet, G ; Bertone, G ; Eggenberger, P
}

\begin{abstract}
We study the effect of dark matter (DM) particles in the Sun, focusing, in particular, on the possible reduction of the solar neutrinos flux due to the energy carried away by DM particles from the innermost regions of the Sun, and to the consequent reduction of the temperature of the solar core. We find that in the very low-mass range between 4 and $10 \mathrm{GeV}$, recently advocated to explain the findings of the DAMA and CoGent experiments, the effects on neutrino fluxes are detectable only for DM models with a very small, or vanishing, self-annihilation cross section, such as the so-called asymmetric DM models, and we study the combination of DM masses and spin-dependent cross sections which can be excluded with current solar neutrino data. Finally, we revisit the recent claim that DM models with large self-interacting cross sections can lead to a modification of the position of the convective zone, alleviating or solving the solar composition problem. We show that when the "geometric" upper limit on the capture rate is correctly taken into account, the effects of DM are reduced by orders of magnitude, and the position of the convective zone remains unchanged.
\end{abstract}

DOI: https://doi.org/10.1103/PhysRevD.82.083509

Posted at the Zurich Open Repository and Archive, University of Zurich ZORA URL: https://doi.org/10.5167/uzh-41578

Journal Article

Accepted Version

Originally published at:

Taoso, M; Iocco, F; Meynet, G; Bertone, G; Eggenberger, P (2010). Effect of low mass dark matter particles on the Sun. Physical Review D, 82(8):083509-14pp.

DOI: https://doi.org/10.1103/PhysRevD.82.083509 


\title{
Effect of low mass dark matter particles on the Sun
}

\author{
Marco Taoso ${ }^{1}$, Fabio Iocco ${ }^{2}$, Georges Meynet $^{3}$, Gianfranco Bertone ${ }^{2,4}$, Patrick Eggenberger ${ }^{3}$ \\ ${ }^{1}$ IFIC (CSIC-Universitat de Valencia), Ed.Instituts, Apt.22085, 46071 Valencia, Spain \\ 2 Institut d'Astrophysique de Paris, UMR 7095-CNRS, \\ Université Pierre et Marie Curie, 98 bis Boulevard Arago 75014, Paris, France \\ 3 Geneva Observatory, University of Geneva, Maillettes 51, 1290 Sauverny, Switzerland and \\ 4 Institute für Theoretische Physik, Universität Zürich, \\ Winterthurerstrasse 190, CH-8057 Zürich, Switzerland
}

\begin{abstract}
We study the effect of dark matter (DM) particles in the Sun, focusing in particular on the possible reduction of the solar neutrinos flux due to the energy carried away by DM particles from the innermost regions of the Sun, and to the consequent reduction of the temperature of the solar core. We find that in the very low-mass range between 4 and $10 \mathrm{GeV}$, recently advocated to explain the findings of the DAMA and CoGent experiments, the effects on neutrino fluxes are detectable only for DM models with very small, or vanishing, self-annihilation cross section, such as the socalled asymmetric DM models, and we study the combination of DM masses and Spin Dependent cross sections which can be excluded with current solar neutrino data. Finally, we revisit the recent claim that DM models with large self-interacting cross sections can lead to a modification of the position of the convective zone, alleviating or solving the solar composition problem. We show that when the 'geometric' upper limit on the capture rate is correctly taken into account, the effects of $\mathrm{DM}$ are reduced by orders of magnitude, and the position of the convective zone remains unchanged.
\end{abstract}

PACS numbers:

\section{INTRODUCTION}

The identification of Dark Matter (DM) is one of the most important open problems of modern cosmology and particle physics. Among the many DM candidates proposed in the literature, Weakly Interacting Massive Particles (WIMPs) are the most popular, as they naturally achieve the appropriate relic density and naturally arise in extensions of the Standard Model of particle physics that will soon be probed in accelerators. WIMPs are currently searched for in a variety of experiments (see Ref. [1] for recent reviews on particle DM, including a discussion of ongoing direct, indirect and accelerator searches).

Here, we focus on the effect of WIMPs on stars, and more specifically on the Sun. In general, WIMPs crossing a star can in principle scatter off baryons to velocities lower than the star escape velocity and be therefore gravitationally trapped by the celestial body, the efficiency of the process depending on the WIMPs scattering cross section and WIMPs ambient density. Once captured, WIMPs can still scatter with the star nuclei and therefore transfer energy inside the object. In addition, WIMPs can eventually annihilate, providing therefore an exotic source of energy. These effects have been investigated in the past, in particular in the context of WIMPs 'cosmions' in order to solve the solar neutrino problem $[2,3]$. This solution has then been discarded in favour of neutrino oscillations and the combination of the cosmion scattering cross-sections and masses suggested by the solar neutrino problem is nowadays excluded by direct detection constraints.

Still, the intriguing possibility that WIMPs modify the properties of the stars has recently prompted new interest on the subject. Recent works have focused in particular on compact objects $[4,5]$, main sequence stars at the Galactic center $[6,7]$ and first stars [8-17]. In all these studies the celestial objects are placed in environments with high DM density, thus enhancing the numbers of WIMPs captured by the star and therefore the WIMPs transport and annihilation energy budgets. However, in these scenarios it is not possible to set robust constraints on the DM cross sections and mass because of the large uncertainties on the DM densities and/or the lack of precise enough observations of the star targets.

The situation is quite different for the Sun since its properties have been measured with good precision. Because of that, the Sun can be used as a diagnostic tool to test small modifications of its structure induced by DM. The most recent works in this direction have focused on modifications of the solar neutrino fluxes and helioseismology data [18-20]. Here we perform a complete and self-consistent calculation of the Sun evolution inside the galactic DM halo. In particular we extend previous works considering light DM candidates, with masses in the range suggested by DAMA [21] CoGent [22] and CDMSII [23] experiments. We consider both standard annihilating WIMPs models and scenarios with negligible DM annihilations. We then move on Self Interacting Dark Matter (SIDM) models [24], which has recently been invoked as a solution of the solar composition problem [25]. Finally, we focus on Inelastic DM models [26].

The paper is organised as follows: in Section II we introduce the DM models used in our calculations. In Section III we present the GENEVA stellar evolution code. In Sec IV we discuss how the solar neutrino fluxes can be used to constrain the DM parameter space. We present our results for standard annihilating WIMPs in Section $\mathrm{V}$, for asymmetric DM models in Section VI, for SIDM 
models in Section VII and for Inelastic DM in Section VIII. Finally we summarize our conclusions in Section IX. Technical details and the formulas implemented in the code are in Appendix A.

\section{THEORETICAL SETUP AND NUMERICAL CODE}

Galactic WIMPs inevitably stream through any celestial object; those which scatter with a nucleus of the celestial object (in the following we will limit ourselves to consider stars) loose energy in such scatter, and if the energy loss brings the velocity of the WIMP below the escape velocity, the WIMP is "captured", i.e. it becomes bound to the star. The formalism to compute the rate at which WIMPs are captured by a star has been object of extensive studies in the 80 's, and here we adopt the established results from [27]. The evolution of the total number of WIMP particles $N_{\chi}$ inside the Sun (or any celestial object) can be written as

$$
\dot{N}_{\chi}=C-2 A N_{\chi}^{2}-E N_{\chi}
$$

where $\mathrm{C}$ is the particle capture rate over the Sun, $\mathrm{A}$ is the annihilation rate and $\mathrm{E}$ the evaporation one. We discuss the details of the time evolution of the WIMP number density in the Appendix A, and we limit ourselves here to discuss the parameters entering in the calculation and the implementation of the theoretical setup in the GENEVA numerical code for stellar evolution.

\section{A. WIMP parameters}

a. DM density. The number of WIMPs captured by a star depends on the WIMP scattering cross section off nuclei, ambient density and velocity distribution (see the Appendix for a detailed discussion). The DM density distribution in the inner regions of the Galaxy is poorly constrained. Semi-analytical studies and simple extrapolations of the results of N-body simulations predict high DM densities at the center of galactic halos, therefore suggesting stars at the galactic center $[6,7]$ and at the center or primordial halos [9-17] as promising targets to detect the effects of WIMPs on stars.

In this paper we focus on the Sun, which being $\sim 8$ $\mathrm{kpc}$ distant from the galactic center, is expected to be placed in a much lower DM density environment than the galactic center. Recent studies [28-30] have shown that current observations constrain the local DM density, $\rho_{\chi}$, within a factor two, assuming spherical DM profiles, while significant deviations may occur for oblate DM distributions. In the following, we adopt $\rho_{\chi}=0.38$ $\mathrm{GeV} \mathrm{cm}{ }^{-3}$, the value obtained in [28] for the benchmark Navarro-Frenk-White density profile. This value was obtained under the assumption that the DM profile is spherically symmetric, while recent numerical simulations that take into account the effect of baryons on the DM density profile suggest that the true DM density is at least $\sim 20 \%$ higher, and that it is affected by large systematic uncertainties due to the unknown position of the Sun in the triaxial DM potential of the Milky Way [31]. Furthermore, the presence of a DM disc, corotating with stars and henceforth "colder" with respect to the solar motion, could enhance the capture rate in the Sun up to a factor ten [32]. Our conclusions can be trivially rescaled for these cases, which however we do not take into account for sake of conservativeness.

b. WIMP-nucleon scattering cross section. The WIMP scattering cross section off nuclei is constrained by direct detection experiments. The strongest bounds on Spin Indipendent (SI) interactions are currently set by CDMS-II [23], XENON100 [33] and CoGeNT [22] which impose $\sigma_{S I} \leq 310^{-40}-410^{-42} \mathrm{~cm}^{2}$ in the mass range $m_{\chi} \sim 5-10 \mathrm{GeV}$. The strongest limit on Spin Dependent (SD) interactions is from PICASSO [34]: $\sigma_{S D} \leq 410^{-36}-410^{-37} \mathrm{~cm}^{2}$ in the same mass range. An evidence for an annual modulation signal has been reported by the DAMA collaboration and subsequently confirmed by the upgraded apparatus $[21,35,36]$. A simple interpretation of this signal in terms of WIMP elastic scattering off nuclei of the target material can hardly be reconciled with the constraints from the other DM detection experiments. In this framework, in fact, the bulk of the region of the $\sigma-m_{\chi}$ parameter space favored by the DAMA signal is excluded by the null results of the other experiments, both considering SI and SD interactions.

Still, there remain small surviving regions of the parameter space for low WIMP masses, $m_{\chi} \sim 10 \mathrm{GeV}$. We address the reader to specific studies for more details (e.g. $[37,38]$ and references therein). Recently, two experiments, CDMSII [23] and CoGeNT [22], have reported an excess in their recoil energy spectra, even if the statistical significance of these signals is very weak. Interestingly, if interpreted in terms of elastic WIMPs scattering, these results would point to light WIMPs, with masses and scattering cross-sections close to the DAMA region $[38,39]$. This scenario has been challenged by the very recent upper limits on SI interactions obtained by XENON100 [33]. However, there are subtle experimental effects that may cast some doubts on the low-mass end of this result, and as we write, the debate between the CoGent and Xenon collaborations is still open. Since the WIMP energy transport inside the star is indeed enhanced for light WIMPs, in Sec.VI we investigate the effects of these particles captured by the Sun on its structure and observables.

\section{B. Dark matter models}

c. Standard WIMPs. Standard WIMPs achieve the DM abundance inferred from cosmological observations for $\langle\sigma v\rangle \sim 3 \times 10^{-26} \mathrm{~cm}^{3} \mathrm{~s}^{-1}$. Although significant deviations from this value are expected for example in presence 
of Sommerfeld enhancements, efficient coannihilations or for non standard cosmologies (see Ref. [1] for more details and references), deviations of few orders of magnitude from the value quoted above are not relevant for the effects of the WIMPs in the Sun, as we shall see in Sec. A. Indeed, the WIMPs annihilation and energy transport are independent from $\langle\sigma v\rangle$ once an equilibrium between scatterings and annihilations is reached. This occurs within a timescale larger than the age of the Sun only for $\langle\sigma v\rangle \lesssim 10^{-33} \mathrm{~cm}^{3} \mathrm{~s}^{-1}$, after Equation A7. Therefore, for the rest of the paper we fix $\langle\sigma v\rangle=3 \times 10^{-26} \mathrm{~cm}^{3} \mathrm{~s}^{-1}$ when we consider annihilating WIMPs models.

d. Asymmetric dark matter DM annihilations can be neglected either in models with very small or vanishing annihilation cross sections or in presence of an asymmetry in the DM sector between particles and antiparticles. This possibility, realized in the so-called asymmetric DM models, is particularly interesting since these DM candidates can in principle have weak-scale interactions and therefore sizable scattering cross sections off baryons, despite they do not annihilate. Concrete realizations of this idea are for example models where the dark sector contains a conserved $U(1)_{X}$ symmetry, analogue of the baryon number, responsible for the stability of the lightest particle in the DM sector. If this quantum charge is shared between baryons and DM it can link the asymmetries in the two sectors and this may naturally explain why the baryons and DM abundances are of the same order of magnitude (see e.g.[40-44]). As noticed above, all DM models for which, the value of the annihilation cross section implies an equilibrium time $\tau_{\odot}>t_{\odot}$, namely $\langle\sigma v\rangle \lesssim 10^{-33} \mathrm{~cm}^{3} \mathrm{~s}^{-1}$, can be considered asymmetric from the point of view of WIMPs accretion.

e. Self-Interacting dark matter. In the scenarios described before, the capture of WIMPs in the star is induced by the interactions of DM with the nuclei. In addition to that, also the elastic scattering of star crossing WIMPs off previously captured WIMPs can contribute to the DM capture rate. For WIMPs self-interactions of the order of the WIMPs scattering cross sections of baryons, like in the models previously considered, this contribution is completely negligible since the number of target nuclei in the Sun is much higher than the number of trapped WIMPs. However, this is not longer true for enhanced self interactions, as predicted in Self Interacting Dark Matter (SIDM) models. In Ref. [45] it has indeed been shown that for extreme values of self interaction cross sections and for $\langle\sigma v\rangle \leq 10^{-27} \mathrm{~cm}^{3} \mathrm{~s}^{-1}$, the annihilation rate can indeed be boosted by a factor of order $\mathcal{O}(100)$, consequently enhancing the prospects for detections of high energy neutrinos produced by WIMPs annihilations in the Sun. SIDM models have been proposed in Ref. [24] to solve some discrepancies of the collisionless Cold Dark Matter (CDM) model, in particular the excess of substructures found in N-body simulations of CDM halos with respect to observations and the conflict between the predicted cuspy CDM density profiles and the observed cored halos of LSB galaxies. Concrete par- ticle physics realizations of SIDM models includes theories with strongly interacting dark sector [42-44], Q-balls $[24,46]$, quark-gluino bound states $[47,48]$ and mirror DM models [49-52]. Observations of galactic and galaxy clusters halos constraint the size of DM self interactions. Here we report the bound obtained from the bullet cluster as a robust upper limit on the SIDM self interaction cross sections [53] : $\sigma_{\chi \chi} / m_{\chi}<2 \times 10^{-24} \mathrm{~cm}^{2} \mathrm{GeV}^{-1}$. We address the reader to Ref. [54] for an updated discussion on the constraints on SIDM.

In the following, since the main focus of the paper is the effect of WIMPs on the Sun, all stellar quantities we show are snapshots taken at the age of the Sun.

\section{THE NUMERICAL CODE}

In order to perform an accurate analysis of the problem we have implemented the capture, annihilation and energy transport of WIMPs in the GENEVA code (for a description of this code, see [55]).

The implementation was done in the following way: from the structure of the stellar model, we can compute at each time step the number of WIMPs in the stellar model, the energy released by annihilation and the energy transferred by the WIMPs. At their turn, these energies modify the structure of the model. Thus the problem has to be resolved self-consistently. To do that, the rate of WIMPs annihilation and transferred energies are added in the equation describing the conservation of the radiative energy. The code then, through an iterative procedure, looks for the values of the pressure, temperature, radius and luminosity at different depths in the stellar model which match for the WIMP modified stellar structure equations (usually a stellar model consists of 900 layers from the surface to the centre).

With this numerical tool, we computed the evolution of one solar mass stellar models from the Zero Age MainSequence $(\mathrm{ZAMS})$ until the solar age $\left(4.57 \times 10^{9}\right.$ years $)$ for various prescriptions for the WIMPs energy transport and annihilation. As initial composition we chose the following values: mass fraction of hydrogen, $X=0.72$, of helium, $\mathrm{Y}=0.266$, and of the heavy elements, $\mathrm{Z}=0.014$. The distribution of the heavy elements was taken as given by [56]. We did not include the effects of microscopic diffusion in these models, although this effect would be required for the computation of tailored models for the Sun [e.g. 57]. The inclusion of this effect would however not change the conclusions of the present work, which are deduced from detailed comparisons of models with exactly the same physics except for the inclusion or not of the WIMPs. Since, microscopic diffusion would have to be accounted in both models, it would not change in a significant way the relative effects obtained here. 


\section{DIAGNOSTIC TOOLS}

The modification of the stellar structure produced by the WIMPs induces changes in the frequencies of stellar oscillations modes and in the neutrino fluxes. The first signature can in principle be observed with helioseismic measurements [18-20], however, the neutrino flux is much more sensitive to the variation of temperature and density profile of the innermost regions of the Sun, and hence it is a much more powerful diagnostic tool.

For the standard solar model, Ref. [58] indicates that the ${ }^{8} \mathrm{~B}$ neutrino flux varies as $T^{25}$, therefore a $1 \%$ temperature change will produce a $25 \%$ change in the ${ }^{8} \mathrm{~B}$ neutrino flux. However, as it has been in shown in Ref. [20], this simple scaling law is not valid to describe the peculiar modifications of the temperature profile induced by the WIMPs. Therefore, the use of a stellar code is mandatory to study the effects on DM on the solar neutrino fluxes.

The distribution of WIMPs inside the Sun is crucial to determine the modifications of the neutrino fluxes. Once captured, WIMPs get redistributed inside a small spatial scale, $r_{\chi}$, of the order of $10^{9} \mathrm{~cm} \sim 0.01 R_{\odot}$, for a WIMP mass of $100 \mathrm{GeV}\left(r_{\chi}\right.$ scales as $m_{\chi}^{-1 / 2}$, see Appendix A 2$)$. The WIMPs energy transport will be therefore more efficient in the innermost regions of the Sun core, as it can be appreciated in the left panel of Fig. 2 (see next sections for more details.) The ${ }^{8} \mathrm{~B}$ and ${ }^{7} \mathrm{Be}$ neutrinos, which are mostly produced at $\sim 0.04 R_{\odot}$ and $\sim 0.06 R_{\odot}$, will be more affected by the presence of WIMPs than the $p p$ neutrinos. In fact, although the $p p$ neutrinos are the most abundant solar neutrinos, they are mainly produced at $\sim 0.1 R_{\odot}$, thus is a region well outside the one affected by the WIMP energy transfer. For the same reason and considering the experimental uncertainties in the determination of ${ }^{8} \mathrm{~B}$ and ${ }^{7} \mathrm{Be}$ neutrino fluxes, we conclude that the ${ }^{8} \mathrm{~B}$ neutrino flux is the best diagnostic tool in order to test the effects of WIMPs on the Sun.

The ${ }^{8} \mathrm{~B}$ flux has been determined with good accuracy by SNO [59]:

$$
\phi_{B}^{\nu}=5.046_{-0.152}^{+0.159}(\text { stat })_{-0.123}^{+0.107} \text { (syst) } 10^{6} \mathrm{~cm}^{-2} \mathrm{~s}^{-1} .
$$

For completeness, we report also the ${ }^{7} \mathrm{Be}$ neutrino flux inferred by Borexino[60]:

$$
\phi_{B e}^{\nu}=(5.18 \pm 0.51) \times 10^{9} \mathrm{~cm}^{-2} \mathrm{~s}^{-1} .
$$

The solar model we use, as described in III, predicts at the solar age $t_{\odot}$ :

$$
\phi_{B}^{\nu}=4.56 \times 10^{6} \mathrm{~cm}^{2} \mathrm{~s}^{-1} \phi_{B e}^{\nu}=4.47 \times 10^{9} \mathrm{~cm}^{-2} \mathrm{~s}^{-1},
$$

values that are well in agreement with the experimental results to within the theoretical uncertainties of solar model calculations [61-63].

Despite its great success in explaining a large variety of observations, the standard solar model suffers nowadays from the so-called solar composition problems. Recent analysis points to a lower surface heavy element content than previously thought (see [64] for recent results) and solar models incorporating these revised metallicities conflict dramatically with helioseismological measurements, in particular right below the solar convective envelope. With the solar abundances of [64], the radius of the convective zone, $R_{C Z}$, is more than $10 \sigma$ higher than the measured value: $R_{C Z}=0.713 \pm 0.001 R_{\odot}$ (see Ref.[61] for an analysis of comparison of different solar models with helioseismology measurements.) High and low heavy elements models also produce differences on $\phi_{B}^{\nu}$ and $\phi_{B e}^{\nu}$ respectively of $\sim 20 \%$ and $10 \%$. However, the theoretical uncertainties obtained within a particular solar model (with high or low metallicity estimations) are significantly smaller, $\sim 10 \%$ and $6 \%$ for $\phi_{B}^{\nu}$ and $\phi_{B e}^{\nu}[62,63]$. Considering the theoretical and experimental uncertainties on $\phi_{B}^{\nu}$, we study the region of the DM parameter space where significant deviations on $\phi_{B}^{\nu}$ are found (the maximum allowed deviation of the ${ }^{8} \mathrm{~B}$ flux depends on the value of the theoretical uncertainties considered. We define this thereshold in Sec.VI). As reference value, we consider the neutrino flux obtained from our solar model without DM particles and in the Sec. VI we define the maximum deviations compatible with present data.

We now discuss the results for standard WIMPs, and provide a description of the role of WIMP energy transport and annihilation. We present separately in the next two sections the results for two special classes of models: asymmetric and self-interacting DM.

\section{RESULTS FOR STANDARD WIMPS}

Since the trapped WIMPs are confined in small spatial scale inside the Sun, the annihilation of WIMPs in the star acts as a point-like source of energy, whose effects have been studied in details in the context of small, and high mass stars, and for various initial metallicities $([6,7,9-16,65])$. When the energy injected through DM annihilation is comparable with the nuclear one, a star finds its equilibrium stage at lower temperatures, thus stopping in a colder region of the Hertzsprung-Russel diagram and prolonging its lifetime. Although the effect is in principle interesting, the required DM densities and cross-sections are such that the effects of an additional energy source due to the DM are totally negligible on the Sun, and anywhere else in the Galaxy but in its innermost regions $[6,7]$. The WIMPs in the star are confined into closed orbits and they inevitably scatter off the stellar material therefore redistributing the energy inside the star. The WIMPs mean free path is $l(r)=1 / \sum_{i} \sigma_{i} n_{i}(r)$, where the sum is performed over all the target nuclei, $\sigma_{i}$ is the WIMP scattering cross section off a certain nucleus and $n_{i}(r)$ is the nucleus number density profile in the star. For reference, the WIMP mean free path is typically of the order $\sim 10^{10} \mathrm{~cm}$ for $\sigma_{S D} \sim 10^{-36} \mathrm{~cm}^{2}$. Once compared with the typical radius of the WIMP cloud $r_{\chi}=10^{9} \mathrm{~cm}$, this indicates that WIMP transport effects are non local. In our computations we span many 

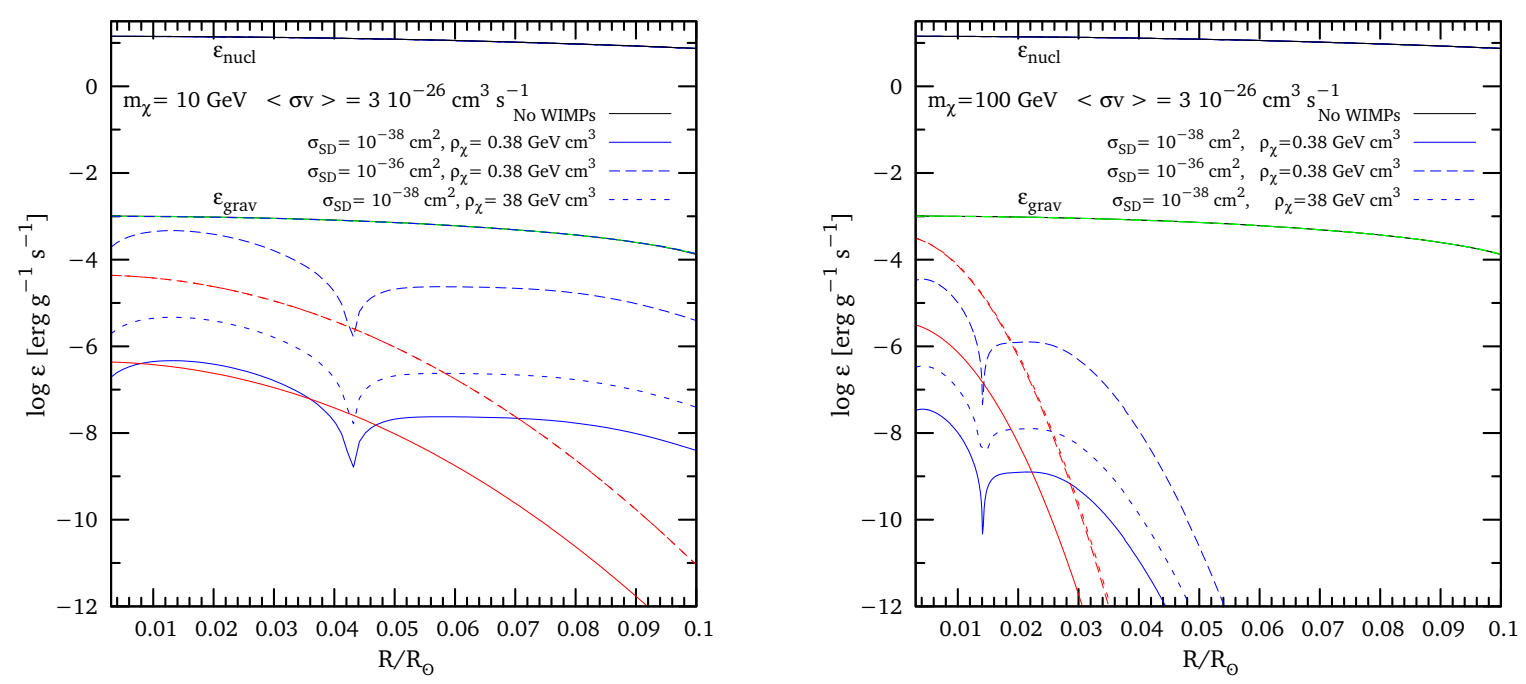

Figure 1: Comparison of the energy transported ( $\epsilon_{\text {trans }}$, blue lines) and injected (red) per unit mass and unit time by WIMPs in the Sun, with the nuclear $\epsilon_{n u c l}$ and gravitational $\epsilon_{\text {grav }}$ energy density of the Star, for $m_{\chi}=10 \mathrm{GeV}$ (left panel) and $m_{\chi}=100$ $\mathrm{GeV}$ (right). Here we plot the absolute value of $\epsilon_{\text {trans }}$, the sign being negative at radii smaller than the dips of the blue curves at $\sim 0.04 R_{\odot}\left(\sim 0.014 R_{\odot}\right)$ in the left (right) panel, and positive otherwise. The sign of $\epsilon_{\text {grav }}$ is positive, this meaning a contraction of the star.

orders of magnitude on the WIMP scattering cross sections so we investigate both local and non-local regimes. We address the reader to Ref. [7], and references therein for further details. The equations implemented in the GENEVA code are summarized in the Appendix A for sake of completeness.

In the non local regime regime, WIMPs scatters can efficiently transport the heat from the inside of the stellar out to colder regions, thus operating toward a flattening of the temperature profile, and a consequent readjustement of the entire stellar structure. Raising the WIMP-proton scattering cross section $\sigma_{0}$, the capture rate (modulo the caveat on saturation of the optical limit, see the Appendix A 1) and the WIMPs scattering rate grows, therefore increasing the energy transported by the WIMPs, $\epsilon_{\text {trans }}$. The dip in the behavior of $\epsilon_{\text {trans }}$ in Figure 1 corresponds to the change in sign of $\epsilon_{\text {trans }}$ : at small radii WIMPs are "colder" than the baryons, and acquire energy with their scatters, which they deposit outward. Since the mean free path of WIMPs scales like $l \sim 1 / \sigma_{0}$, at large scattering cross sections the WIMPs remain progressively "trapped" in the interior of the star. This implies that the heat transport from DM particles becomes local and therefore $\epsilon_{\text {trans }}$ dramatically reduces.

The WIMP mass also plays a role in determining the effects on DM in stars, as it can be appreciated in Equations in Appendix A. Lowering the DM mass goes in the direction of maximizing the transport effects, but also the evaporation rate. Above $\mathrm{m}_{\chi}=5 \mathrm{GeV}$, evaporation can be safely neglected and the mass of the particle acts to modify the radius and normalization of DM inside the star.

In Figure 1 we compare the rate of energy released per unit of baryonic mass by DM annihilation $\epsilon_{a n n}$ and the rate of energy absorbed or released by DM transport $\epsilon_{\text {trans }}$, with the rate of energy produced by nuclear reactions. The solid lines represent two benchmark WIMP scenarios for the Sun: a local DM density of $\rho_{\chi}=0.38 \mathrm{GeV} / \mathrm{cm}^{3}$, a Spin-Dependent scattering cross section $\sigma_{S D}=10^{-38} \mathrm{~cm}^{2}$ and WIMP mass and selfannihilation cross-section compatible with a DM thermal production scenario. Since in these computations the WIMP annihilations and transport energies are negligible with respect to the energy provided by nuclear reactions, the structure of the Sun is not affected by the presence of the WIMPs. As a consequence of that, the solar neutrino fluxes are unchanged with respect to their values in our standard solar model without DM particles. As stated before, the WIMP energy transport is more efficient for light WIMPs: in Figure 1 it dominates over the annihilation energy for a $10 \mathrm{GeV}$ WIMP while the opposite trend occurs for a $100 \mathrm{GeV}$ WIMP, at least in the inner region of the Sun. However, due to the different scaling of $\epsilon_{\text {ann }}$ and $\epsilon_{\text {trans }}$ with $\rho_{\chi}$, at high DM densities the annihilation energy is always the most relevant source of energy produced by the WIMPs in the star. An example of that is given by the dashed curve in Figure 1, where we raise the DM density of two orders of magnitude, $\rho_{\chi}=38 \mathrm{GeV} / \mathrm{cm}^{3}$.

Rescaling our curves in Fig. 1 using Eqs. 1, A3 and A6, it can be noticed that energy injection by DM annihilation is comparable with the nuclear energy for DM densities of order $\rho_{\chi} \sim 10^{8} \mathrm{GeV} / \mathrm{cm}^{3}$. This value is indeed the typical DM density for which the behaviour of main sequence stars starts to be severely modified by WIMPs annihilations $[6,7]$. 

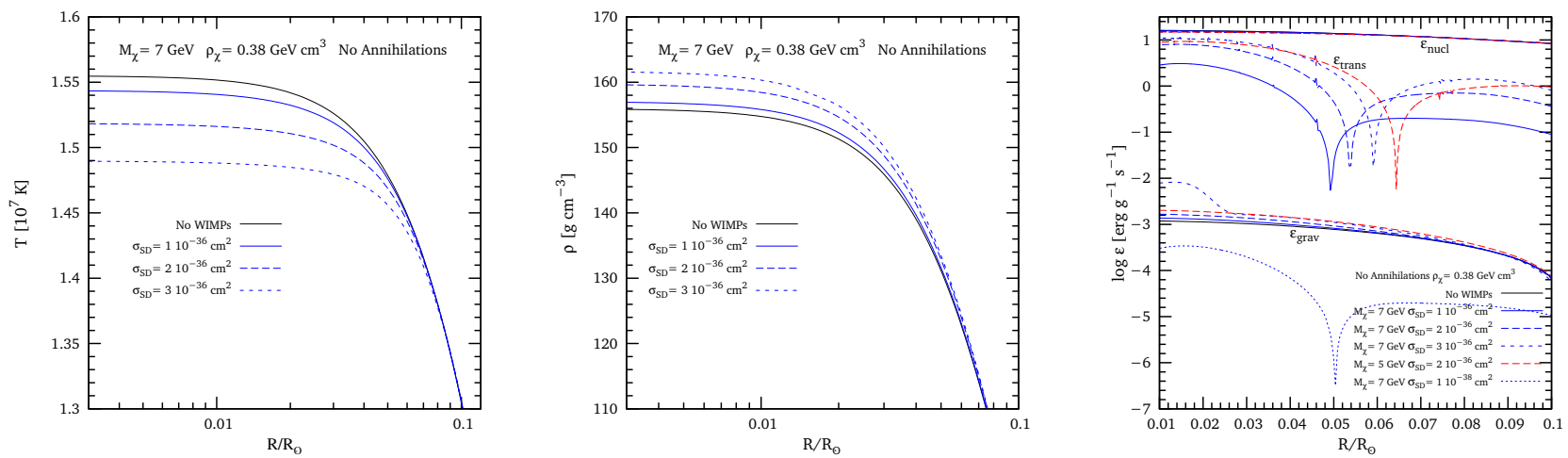

Figure 2: Impact of asymmetric DM on the Sun. Left Panel: Temperature profile of the Sun for different SD scattering cross-sections at $t_{\odot}=4.57$ Gyrs. Central Panel: Density profile (baryons only) inside the Sun. Right Panel: Energy transport (meaning of the curves as in Fig.1). The sign of $\epsilon_{\text {grav }}$ is positive.

In conclusion, the Sun's global properties are not dramatically changed by self-annihilating WIMPs, and no solar observable can be used as a good diagnostic for these models. As we explain in the following Sections, things are quite different for other DM scenarios.

\section{RESULTS FOR ASYMMETRIC DM}

If WIMPs are asymmetric, Equation 1 reads:

$$
\dot{N}_{\chi}=C-E N_{\chi}
$$

and particle will continue accumulating in the center of the star, since their abundance will not be limited by annihilation.

As before, DM particles rapidly thermalize, therefore the shape of the WIMPs distribution stays as in the annihilating case whereas the normalization $N_{\chi}$ will result modified. The number of WIMPs inside the Sun, neglecting evaporation, will simply be $N_{\chi}=C t_{\odot}$, with $t_{\odot}$ the age of the Sun. In the annihilating case, after a transient $\tau_{\chi}$, an equilibrium between annihilations and capture is reached and $N_{\chi}$ stays $N_{\chi}=C \tau_{\chi}$. Comparing $t_{\odot}=4.57 \times 10^{9} \mathrm{yr}$ with the typical equilibrum timescale in a vanilla WIMPs scenario, $\tau_{\chi} \sim$ $10^{6} \mathrm{yr}\left(\langle\sigma v\rangle / 10^{-26} \mathrm{~cm}^{3} \mathrm{~s}^{-1}\right)^{-1 / 2}$ for $m_{\chi}=10 \mathrm{GeV}$, it is evident that the number of WIMPs trapped in the Sun is significantly bigger in asymmetric models.

In order to study the effects of asymmetric DM, we have computed the variations of neutrino fluxes produced by the heat transport of WIMPs in the Sun. We evolved the Sun from the ZAMS up to its current age $t_{\odot}$ with the GENEVA stellar code. As previously discussed, the WIMPs effects are accounted for self-consistently at each time-step; if, for instance, energy is evacuated by the WIMPs in the very central layers, the structure will react by allowing the central layers to contract. The present numerical approach is therefore more accurate and self consistent than methods in which the effects of WIMPs are deduced from the structure of standard solar models computed without WIMPs effects, this, for at least two reasons: first because in the present approach the structure of the star can readjust itself to any changes produced by the WIMPs and second because, these readjustments are accounted for during the whole previous nuclear evolution of the Sun.

The first two panels of Figure 2 shows the temperature and density profiles of the Sun in presence of asymmetric DM. Note the change in temperature with increasing SD scattering cross section: the temperature decreases at the center and (although this is difficult to appreciate in the plot) slightly increases close to the external edge of the stellar core. The reason of the decrease in temperature can be understood in terms of energy transported away from the solar core. In general, any amount of energy removed from the core leads to its contraction. Naively, one would expect a warming of the internal regions of the Sun since part of the energy extracted from the gravitational energy reservoir goes into internal energy. However, the energy carried away by the WIMPs is well above the energy released by the core contraction, resulting therefore in a cooling of the central regions.

The right panel in Figure 2 demonstrates in fact that the energy transported by asymmetric DM can become extremely high, and it can significantly modify the structure of the star. We show the results for two WIMP masses: at fixed $\sigma_{S D}$ the normalization of $\epsilon_{\text {trans }}$ is higher for a $5 \mathrm{GeV}$ particle than for a $7 \mathrm{GeV}$ one, and the sign inversion point of the energy transport term shifts outwards. The shift in the sign inversion point between different curves for the same DM mass is due to the feedback induced by the modification of the density profile, which starts becoming sensible at $\sigma_{S D} \approx 10^{-36}$.

In principle one should study how the structural modifications modify the details of neutrino oscillations in the Sun and consequently the inferred values of the neutrino oscillations parameters, i.e. $\Delta m_{\odot}^{2}$ and $\sin \left(\theta_{\odot}\right)$. A sizeable effect is actually expected for density variations 

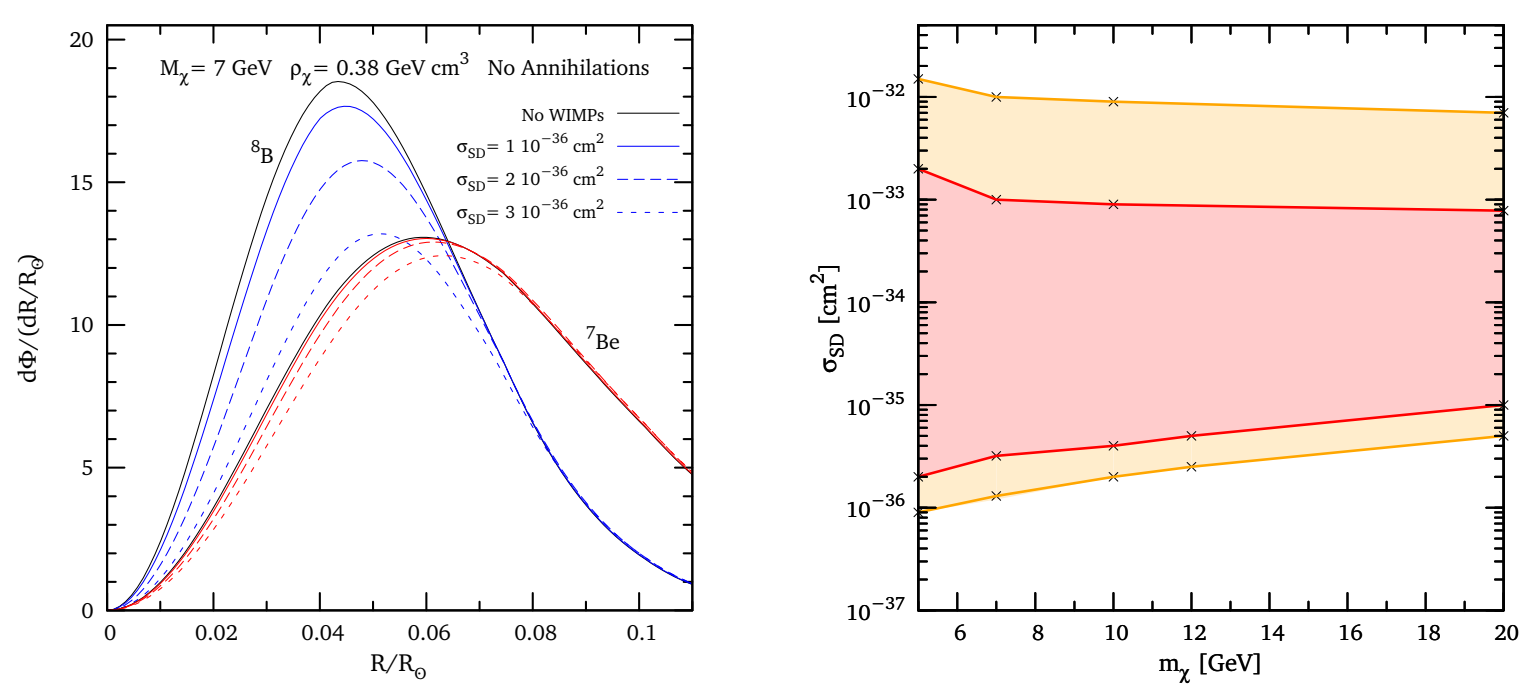

Figure 3: Solar neutrino fluxes in presence of asymmetric DM in the Sun. Left Panel: Differential ${ }^{8} \mathrm{~B}$ and ${ }^{7}$ Be neutrino fluxes as a function of radius. The curves referring to our solar model are normalized to unity. Right panel: isocontours of $25 \%$ (red) and $5 \%$ (yellow) $\phi_{B}^{\nu}$ deviations with respect our solar model prediction in the $m_{\chi^{-}} \sigma_{S D}$ plane. The red area show the region of the parameter space with $\phi_{B}^{\nu}$ modifications larger than $25 \%$ and therefore in tension with present $\phi_{B}^{\nu}$ data. The weakening of the DM transport effects at high cross sections is due to the transition to local transport regime. See text for details.

of the order of $10 \%$ at $\sim 0.04 R_{\odot}$, which corresponds to the region of maximal ${ }^{8} \mathrm{~B}$ production [66]. However, at that radii, we find a decrease of the density below $2 \%$ even for models producing modifications of the $\phi_{B}^{\nu}$ larger than $20-25 \%$. This modification is comparable with the uncertainties on the density profile in the inner regions of the Sun, which is of the order of $1 \%$ for $R<0.45 R_{\odot}$ [67].

These considerations are valid also for the ${ }^{7}$ Be fluxes and therefore modifications of the neutrino oscillation probabilities are completely negligible for our purposes.

Left panel of Figure 3 shows the differential ${ }^{8} \mathrm{~B}$ and ${ }^{7} \mathrm{Be}$ neutrino fluxes as a function of the stellar radius, in presence of different WIMP models. As expected, the reduction of the neutrino production, due to the cooling of the baryons inside the Sun, is more efficient at small radii, where the WIMPs are concentrated. As noticed in Sec. IV, this leads to a larger modification on the total ${ }^{8} \mathrm{~B}$ neutrino flux, $\phi_{B}^{\nu}$, which is the integral over the whole Sun of the corresponding differential quantity plotted in the left panel of Figure 3, than those on the ${ }^{7}$ Be neutrino flux, $\phi_{B e}^{\nu}$.

To study the impact of these structural variations on the solar neutrino flux, we have performed a systematic study of the DM parameter space, varying the WIMPs scattering cross section and mass. For SI interactions we find sensible variations of the neutrino fluxes only for very large values of $\sigma_{S I}$, already severely excluded by direct detection experiments, therefore we focus for the rest of the section on SD interactions.

In the right panel of Fig. 3, we show in the $m_{\chi}-\sigma_{S D}$ plane the isocontours corresponding to $\phi_{B}^{\nu}$ variations of $25 \%$ and $5 \%$ with respect to our solar model without
WIMPs. For masses below $4-5 \mathrm{GeV}$ the evaporation becomes important and the number of WIMPs inside the star is strongly suppressed.

Above $m_{\chi}=20 \mathrm{GeV}$ the WIMP transport starts to become inefficient and even for high scattering cross section (still in the non-local transport regime) the WIMP energy transport is non negligible only at the very center of the star, providing a local dip of energy. However, for increasing WIMPs masses the existing constraints from direct detection experiments become more severe, and the region of the parameter space able to produce sizeable modifications of $\phi_{B}^{\nu}$ is already excluded. Because of that, we do not explore that region any further. At very high scattering cross sections (i.e. $\gtrsim 10^{-33} \mathrm{~cm}^{2}$ ) the WIMPs heat transport becomes more and more localised and the modifications of $\phi_{B}^{\nu}$ tend to decrease, consistently with what is described in Sec.V. This is also the reason of the non specularity of the exclusion curves in Figure 3: the low and high cross-section regions are characterized by different physics (non-local vs local transport effects, respectively).

Combining experimental and conservative (20\%) theoretical uncertainties we derive that modifications of the $\phi_{B}^{\nu}$ above $\sim 30 \%$ are excluded at $95 \% \mathrm{CL}$. The maximum modifications that we obtained in our computations are slightly below this level: further increasing $\sigma_{S D}$, problems are encountered in solving the stellar structure at ages well below the solar one. We have not further investigated if these difficulties are merely a numerical artifact or are instead related to the non existence of a solution of the stellar structure. However, we notice that once we obtain variations of $\phi_{B}^{\nu}$ of the order of $20 \%$, further small changes of $\sigma_{S D}$ induce rapid modifications of $\phi_{B}^{\nu}$. 
Because of that, the isocontours corresponding to $30 \%$ $\phi_{B}^{\nu}$ variations should be closed to the ones corresponding to $\delta \phi_{B}^{\nu}=25 \%$ shown in Fig. 3 .

Considering a more optimistic value for the theoretical uncertainties on the $\phi_{B}^{\nu}$ predictions, i.e. $10 \%$, the threshold for exclusion at $95 \%$ CL is lowered to $18 \%$ variations from our solar model prediction. Most of the SD crosssections inside the $25 \%$ region in Fig. 3 are excluded by the direct detection experiments constraints, apart from a small region at low masses which is somewhat in tension with those bounds. A reduction of the theoretical and experimental uncertainties on $\phi_{B}^{\nu}$ in the next years may in principle improve the sensitivities on $\sigma_{S D}$. We show however in Fig.3 that considering a $5 \%$ modification of $\phi_{B}^{\nu}$ the region of the $m_{\chi}-\sigma_{S D}$ parameter space which can be probed enlarges very little. Our findings are qualitatively consistent with previous results $[3,20]$.

\section{RESULTS FOR SELF-INTERACTING DM}

As we have seen, since DM annihilations limit the number of trapped WIMPs in the Sun, asymmetric DM models are promising scenarios to look for modifications of the Sun properties. This is particularly true for the case of SIDM models, as it has been noticed in Ref. [25]. In presence of only DM self-interactions and DM scattering off baryons, the number of DM particle inside the Sun is given by:

$$
\dot{N}_{\chi}=C+C_{\chi \chi} N_{\chi}
$$

where $C_{\chi \chi}$ is the DM capture rate through DM self interactions. The solution of this equations reads:

$$
N_{\chi}(t)=\frac{C}{C_{\chi \chi}}\left(e^{C_{\chi \chi} t}-1\right)
$$

and reduces to $N_{\chi}(t)=C t$ for negligible self interactions, i.e. the formula used in Sec.VI It can be noticed therefore that the numbers of captured DM particles is exponentially enchanced in asymmetric SIDM models. [45]:

The self-capture rate $C_{\chi \chi}$ has been obtained in Ref

$$
C_{\chi \chi}=\sqrt{\frac{3}{2}} \rho_{\chi} \frac{\sigma_{\chi \chi}}{m_{\chi}} \frac{v^{2}(r)}{\bar{v}}\left\langle\phi_{\chi}\right\rangle \frac{\operatorname{Erf}(\eta)}{\eta} .
$$

where the average of $\phi(r) \equiv v^{2}(r) / v^{2}\left(R_{*}\right)$ over the WIMPs distribution gives $\left\langle\phi_{\chi}\right\rangle \simeq 5.1$. $R_{*}$ refers to the Sun radius and the definition of the other quantities can be found in Appendix.

An upper bound to the total WIMPs self-interaction arises when the sum of the short range WIMPs selfinteraction cross sections over all the WIMPs targets equals the surface of the WIMPs distribution:

$$
\sigma_{e f f, \chi} \equiv \sigma_{\chi \chi} N_{\chi}=\pi r_{\chi}^{2}
$$

In the equation above, we can safely consider that all the WIMPs are localized inside the thermal readius $r_{\chi}$. Once the geometrical limit is saturated at a time $\hat{t}$ with a number of WIMPs $N(\hat{t})$ inside the star, we simply replace the combination $\sigma_{\chi \chi} N_{\chi}$ in Eq.3 with $\sigma_{e f f, \chi}$. The WIMPs number density for $t>\hat{t}$ is therefore given by

$$
\hat{N}_{\chi}(t)=\left(C+\hat{C}_{\chi \chi}\right)(t-\hat{t})+N(\hat{t})
$$

with $\hat{C}_{\chi \chi}$ obtained replacing $\sigma_{\chi \chi}$ in Eq. 5 with $\sigma_{e f f, \chi}$.

We have studied the effect of a population of SIDM models in the Sun, implementing in the GENEVA code equations $3-5$. To exemplify our results we focus on a DM mass $m_{\chi}=7 \mathrm{GeV}$ and we consider $\sigma_{S I}=2 \times 10^{-41} \mathrm{~cm}^{2}$ and $\sigma_{S D}=10^{-36} \mathrm{~cm}^{2}$, i.e. values close to the bounds set by direct detection constraints. In Fig. 4 we show the evolution in time of the number of captured WIMPs normalized to the total number of baryons, $N_{\chi} / N_{\odot}$ for the two models here considered and for a self-interaction cross sections which saturates the bound from the bullet cluster mentioned above. In the case of a pure SI interaction it can be noticed the change between the exponential rise regime and the linear one, occurring when the geometrical limit is saturated. For SD interactions the same happens but since the geometrical bound is obtained earlier in the evolution this modification is difficult to notice. In both calculations we have not found any significant deviation of the solar structure at the Sun age. For the case of SD interaction, the WIMPs transport energy $\epsilon_{\text {trans }}$ is at most a factor 2 smaller than the nuclear energy. Small radiative opacity variations occur only at $r \lesssim 0.05 R_{\odot}$. These small effects does not lead to any significant variations of the sound speed profiles in the whole Sun, and so of $R_{C Z}$.

In Ref.[25], adopting a polytropic model for the Sun structure and the linearized solar model of Ref. [68] the authors concluded that for suitable values of SIDM parameters the boundary of the solar convective zone $R_{C Z}$ is decreased. They claimed that these modifications are such that the solar composition problem mentioned above can be solved, i.e. the position of the convective zone and helioseismology data can be reproduced.

Our analysis does not confirm their conclusions. In order to maximize the WIMP energy transport, we have artificially turned off the geometrical bound in our code and evolved different solar models. We stress however that this scenario is unphysical. We have studied both models with pure SD and pure SI interactions, leaving the DM mass and cross section at the values fixed before. We have then varied the DM self-interaction crosssection in order to maximize the number of SIDM in the Sun. In the extreme cases we have found a dramatical reduction of the core temperature, which produces a significant decrease of $\phi_{B}^{\nu}$, in agreement with what is obtained in Sec. VI. At the center of the star, the luminosity is reduced, due to the evacuation of energy produced by the WIMPs, and the radiative opacity is increased, as a result of the decrease of the temperature and the increase 


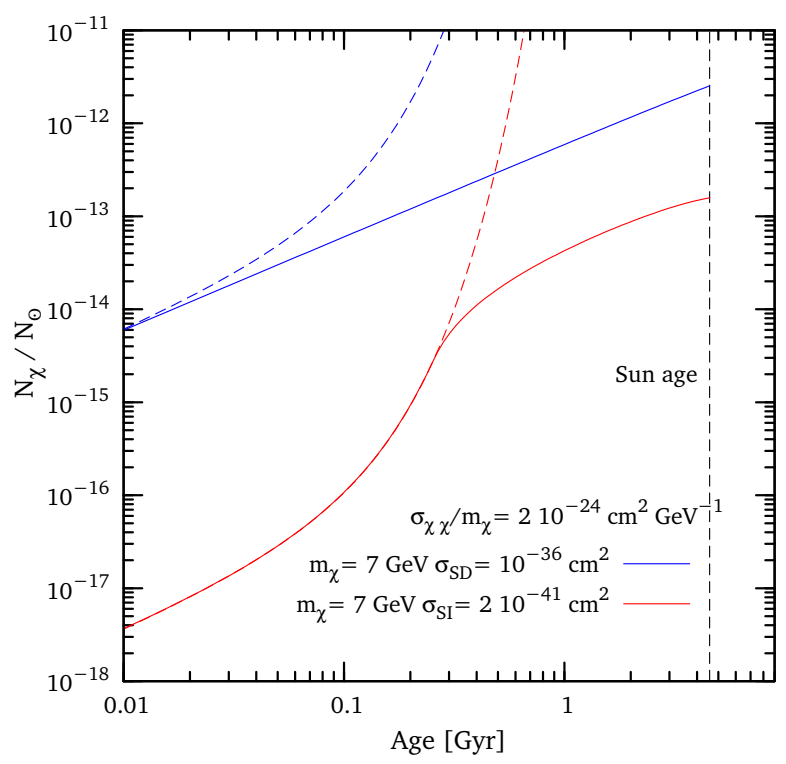

Figure 4: Number of WIMPs inside the star normalized to the number of baryons as a function of the age in the asymmetric SIDM scenario. Solid and dashed lines refer to calculations which respectively implement or neglect the geometrical bound on the self interaction.

of the density. The external zones are not significantly affected by the WIMPs so we do not see any significant change in the position of the convective zone. We conclude that a population of WIMPs, being strongly localized at the center of the Sun, can not affect its external shells without at the same time completely change the internal structure of the star. On the other hand, strong modifications of the central solar structure traduce in dramatic changes on the solar neutrino fluxes and helioseismology g-modes, as also demonstrated in Ref. [69], and in general of any observable sensitive to the physical conditions in that inner regions. The results of Ref. [69] appear in good agreement with our own and confirm that the presence of WIMPs inside the Sun can not modify the solar sound speed profile in such a way to restore the agreement with helioseismological data.

\section{INELASTIC DM}

The possibility that DM particles could scatter inelastically up to an excited state, has been recenly proposed in the literature in the framework of theoretically justified models [26, 70-72], in order to explain the modulation observed in the DAMA signal. The existence of two states splitted by an energy $\delta$ introduces a threshold for the WIMPs scattering since only WIMPs with enough kinetic energy, $E_{k}$ can be excited to the heavier state:

$$
E_{k} \geq \delta\left(1+\frac{m_{\chi}}{M_{N}}\right)
$$

with $m_{N}$ the mass of the nuclei. Since the energy threshold depends on the type of nucleus considered, in the inelastic DM scenario the scattering rate can differ dramatically between different targets, opening the possibility to reconcile the DAMA signal with the results of the other experiments [38, 73-76]. Recent analyses favor WIMP masses of the order $m_{\chi} \sim 10-50 \mathrm{GeV}$ and small mass splitting $\delta \sim 30-130 \mathrm{KeV}$. We address the reader to the original literature for more details.

The WIMPs capture rate in the Sun for inelastic DM models tends to be reduced with respect to the elastic case by the presence of the inelastic barrier. However, since a significant fraction of the kinetic energy lost by the WIMPs goes into excitation, less energy is transferred to the nuclei, thus reducing the nucleus form-factor suppression. Explicit calculations have shown than, for certain choices of the DM parameters, the capture rate can be enhanced up to an order of magnitude [77, 78]. Another important effect of the existence of the energy threshold $\delta$ for scatter is the modification of the internal distribution of DM particles inside the star: as a consequence of the reduced number of scatters, they can't shed away their angular momentum entirely, and their orbits remain larger than in a standard, elastic DM case (see Fig 1a in [77]).

Here we aim to understand if inelastic DM models can affect the Sun's properties, and if the diagnostics we have discussed in previous Sections allows to probe this class of models. First, we notice that, once captured, the velocity of DM particles will be smaller than the local escape velocity. Considering an escape velocity at the Sun core of $v=1300 \mathrm{~km} \mathrm{~s}^{-1}$ and taking a WIMPs speed at infinity $u=220 \mathrm{~km} \mathrm{~s}^{-1}$ the maximum kinetic energy of a trapped WIMPs will be $E_{k}^{\max } \sim 96.5 \mathrm{KeV}\left(m_{\chi} / 100 \mathrm{GeV}\right)$. Looking at Eq. 6, it can be realised that once captured, WIMPs can scatter only with elements heavier than hydrogen, even considering a small mass splitting $\delta=30$ $\mathrm{keV}$. Simple kinematic considerations show that after a few scatterings the WIMPs will not have enough kinetic energy to overcome the inelastic threshold. For example, for the same $\delta=30 \mathrm{keV}$, a WIMP with mass $m_{\chi}=100 \mathrm{GeV}$ will scatter in average $\sim 5$ times off iron or off helium. Therefore, at a given time, only a small part of the WIMPs population inside the star can effectively scatter and transport energy. Focusing on the most optimistic asymmetric case, the number of WIMPs above the kinematic thereshold, $N_{\chi}^{\prime}$, at given time can be obtained from:

$$
\dot{N}_{\chi}^{\prime}=C-N_{\chi}^{\prime} \tau_{i n}^{-1}
$$

where $\tau_{\text {in }}$ is the average time needed for a WIMP to fall below the inelastic thereshold. This quantity can be estimate as:

$$
\tau_{i n}=N_{s c} \frac{l}{w}
$$


where $l$ is the WIMP mean free path defined in Sec. V, $w$ is the WIMP velocity inside the star and $N_{s c}$ is the number of WIMP scatterings. Here we take a mean baryon density $\rho \sim 10^{2} \mathrm{~g} \mathrm{~cm}^{-3}$ and we consider only scattering off elements heavier than helium, which in total account for $\sim 1 \%$ of the Sun mass. We obtain therefore:

$$
\tau_{\text {in }}=2.3 \times 10^{5} \mathrm{~s} \frac{N_{s c}}{30} \frac{1318 \mathrm{~km} \mathrm{~s}^{-1}}{w} \frac{100 \mathrm{~g} \mathrm{~cm}^{-3}}{\rho} \frac{10^{-36} \mathrm{~cm}^{2}}{\sigma_{S D}}
$$

At the age of the Sun, the number of WIMPs transporting energy inside the star will be $C \tau_{i n}$, which is many orders of magnitude lower than in the elastic case, $C t_{\odot}$.

Considering the capture rate obtained with the most favored values of inelastic DM parameters, from the results obtained in Sec. VI we conclude that inelastic DM models do not produce any detectable modifications of the Sun properties.

\section{CONCLUSIONS}

By the use of a stellar evolution code, we have studied the modifications on the Sun structure induced by DM particles captured by the Sun during its lifetime. For standard WIMPs an equilibrium between the capture and the annihilation rates is reached in short timescales. After this transient period, the number of WIMPs inside the Sun is constant and we find it to be too small to produce any observable effect on the Sun properties.

However, for scenarios with null or very small annihilation cross sections, $\langle\sigma v\rangle \lesssim 10^{-33} \mathrm{~cm}^{3} \mathrm{~s}^{-1}$, the number of trapped WIMPs is significantly increased. In this case, the transport of energy by the WIMPs from the interior of the Sun to the outer shells can dramatically reduce the core temperature, the most important consequence being a reduction of the ${ }^{8} B$ flux. Considering the present theoretical and experimental uncertainties on $\phi_{B}^{\nu}$, we have studied the combination of DM masses and SD scattering cross sections which can be ruled our with this argument, finding only a small region of the parameter space which is not already excluded by direct detection bounds. Even with a significant decrease of the uncertainties on $\phi_{B}^{\nu}$, the region of the parameter space which can be probed remains approximately the same, therefore future experimental advances will not significantly change the situation.

We have also considered SIDM candidates which have been recently invoked as a solution of the solar composition problem. Correctly implementing the geometrical limit, we find that the number of WIMPs captured on the star is not sufficient to produce significant deviations on the Sun properties, unless one considers very high scattering cross sections, where however the models become in tension with direct detection bounds. Even in this case, we encounter significant modifications of the density and temperature profiles only in the inner regions of the Sun while the outer shells are not significantly affected. We can therefore exclude that the transport of energy produced by a population of WIMPs can solve or even alleviate the solar composition problem.

Finally, we notice that the WIMPs energy transport may produce dramatic effects on the structure of stars settled in high DM density environments, like the galactic center. We leave the investigation of this scenario for future work.

When this manuscript was ready for submission, a similar work appeared on the arXiv, focusing on the effects of WIMPs in the Sun [69]. While our work mainly focus on the modifications of the solar neutrino fluxes, Ref. [69] considers future helioseismology data as a diagnostic tool to constrain the WIMPs parameter space.

\section{Acknowledgments}

We thank N.Fornengo and A.Palazzo for useful conversations. We thank S.Sarkar and M.T.Frandsen for discussions and the comparison of the results. MT acknowledges support of the Spanish MICINNs ConsoliderIngenio 2010 Programme under grant MULTIDARK CSD2009-00064. His work is partly supported by the Spanish grants FPA2008-00319 (MICINN) and PROMETEO/2009/091 (Generalitat Valenciana) and the European Council (Contract Number UNILHC PITN-GA2009-237920). FI acknowledges support from the European Community research program FP7/2007/2013 within the framework of convention \#235878.

\section{Appendix A: Formalism and code}

\section{Capture}

The formalism to compute the rate at which WIMP particles are captured by a star have been extensively studied in the eighties. Here we adopt the results from [27], who gives for the capture rate $C$ :

$$
C=\sum_{i} 4 \pi \int_{0}^{R_{*}} d r r^{2} \frac{d C_{i}(r)}{d V}
$$

with

$$
\begin{aligned}
\frac{d C_{i}(r)}{d V} & =\left(\frac{6}{\pi}\right)^{1 / 2} \sigma_{\chi, N_{i}} \frac{\rho_{i}(r)}{M_{i}} \frac{\rho_{\chi}}{m_{\chi}} \frac{v^{2}(r)}{\bar{v}^{2}} \frac{\bar{v}}{2 \eta A^{2}} \\
& \times\left\{\left(A_{+} A_{-}-\frac{1}{2}\right)\left[\chi(-\eta, \eta)-\chi\left(A_{-}, A_{+}\right)\right]\right. \\
& \left.+\frac{1}{2} A_{+} e^{-A_{-}^{2}}-\frac{1}{2} A_{-} e^{-A_{+}^{2}}-\frac{1}{2} \eta e^{-\eta^{2}}\right\} \\
A^{2} & =\frac{3 v^{2}(r) \mu}{2 \bar{v}^{2} \mu_{-}^{2}}, \quad A_{ \pm}=A \pm \eta, \quad \eta^{2}=\frac{3 v_{*}^{2}}{2 \bar{v}^{2}}
\end{aligned}
$$




$$
\begin{gathered}
\chi(a, b)=\frac{\sqrt{\pi}}{2}[\operatorname{Erf}(b)-\operatorname{Erf}(a)]=\int_{a}^{b} d y e^{-y^{2}} \\
\mu_{-}=\left(\mu_{i}-1\right) / 2, \quad \mu_{i}=m_{\chi} / M_{i}
\end{gathered}
$$

where $\rho_{i}(r)$ is the density profile of a given chemical element in the interior of the star and $M_{i}$ refers to its atomic mass, while $m_{\chi}$ and $\rho_{\chi}$ are respectively the WIMP mass and the WIMP density at the star position. The analytic expression for the capture rate per shell volume reported above, $d C / d V$, is obtained for the case of a MaxwellBoltzmann velocity distribution with speed dispersion $\bar{v}$; throughout this paper, we adopt $\bar{v}=270 \mathrm{~km} \mathrm{~s}^{-1}$, and we take the velocity of the star moving through the DM halo, labeled as $v_{*}$, to be $v_{*}=220 \mathrm{~km} \mathrm{~s}^{-1}$, as appropriate for the Sun. The radial escape velocity profile depends on $M(r)$, i.e. the mass enclosed within a radius $r, v^{2}(r)=2 \int_{r}^{\infty} G M\left(r^{\prime}\right) / r^{\prime 2} d r^{\prime}$. The DM elastic scattering cross section off nuclei $\sigma_{\chi N_{i}}$ is the sum of the Spin-Dependent (SD) and Spin-Independent (SI) contributions:

$$
\sigma_{\chi N_{i}}=\beta^{2} A_{i}^{2} \sigma_{S I}+\beta^{2} \sigma_{S D} \frac{4\left(J_{i}+1\right)}{3 J_{i}}\left|\left\langle S_{i, p}\right\rangle+\left\langle S_{i, n}\right\rangle\right|^{2}
$$

with $\sigma_{S I}$ and $\sigma_{S D}$ the hydrogen-normalized SI and SD nuclear-scattering cross sections. The factor $A_{i}$ is the atomic number while $J_{i}$ is its spin and $\beta$ the ratio of the reduced mass of the WIMP-nucleus and WIMP-proton systems. Finally, $\left\langle S_{i, p}\right\rangle$ and $\left\langle S_{i, n}\right\rangle$ are respectively the expectation values of the spin content of the proton and neutron group in the nucleus. The total capture rate is then obtained summing up all the elements present in the star.

The geometrical size of the star itself sets an upper bound to the effective WIMP scattering crosssection, $\sigma_{\text {eff, }}$ which we model imposing a maximum to the capture rate once the condition below is reached:

$$
\sigma_{e f f} \equiv \sum_{i} \sigma_{\chi N_{i}} N_{i}=\pi R_{\star}^{2}
$$

with $N_{i}$ the number of nuclei of the element $i$; this is sometime referred to as the optical limit.

\section{Annihilation}

Throughout this paper we work under the assumption that WIMPs thermalize inside the Sun within a negligigible timespan with respect to stellar evolutionary timescale. This is a good approximation for most WIMP models, and especially for the high scattering cross-sections we will examine, as an upper limit of the thermalization time reads [13]:

$$
\tau_{t h}=\frac{4 \pi}{2 \sqrt{2 G}} \frac{m_{\chi}}{\sigma_{\chi N_{i}}} \frac{R_{*}^{7 / 2}}{M_{*}^{3 / 2}}
$$

that for the sun means

$$
\tau_{t h} \sim 10^{5} s\left(\frac{m_{\chi}}{10 \mathrm{GeV}} \frac{10^{-36} \mathrm{~cm}^{2}}{\sigma_{\chi N_{i}}}\right) .
$$

In the case of a non-local WIMP energy transport, i.e. for large $K$ (see next section), the WIMPs clould shares a global temperature which can be set, in good approximation, to the star core temperature $T_{c}$. The WIMP radial distribution in the star gravitational well is thus given by [79]:

$$
n_{\chi, n l}(r)=n_{\chi, 0} e^{\frac{-r^{2}}{r_{\chi}^{2}}}, r_{\chi}=\sqrt{\frac{3 k T_{c}}{2 \pi G \rho_{c} m_{\chi}}}
$$

with $\rho_{c}$ referring to the core density. The distribution results quite concentrated toward the center of the star: typical values for ZAMS stars are $r_{\chi} \sim 0.1 r_{c} \sim 10^{9} \mathrm{~cm}$, $r_{c}$ being the stellar core radius. Such zero order distribution is slightly modified by transport effects, see Section A 4, and we adopt the modified distribution to compute the actual rate of annihilations; however, the effects of such modification are small and their effects on annihilations negligible in practice. Once the normalization $n_{\chi, 0}$ is obtained solving the Eq.1, the distribution $n_{\chi}(r)$ is completely specified and the annihilation term can be easily computed:

$$
A=\int_{0}^{R_{\odot}} \epsilon_{a n n} r^{2} 4 \pi \rho(r) d r,
$$

with:

$$
\epsilon_{\text {ann }}=\frac{1}{2}\langle\sigma v\rangle m_{\chi} c^{2} n_{\chi}^{2}(r) \rho(r)^{-1} .
$$

$\rho(r)$ is the baryon density at a given position and $\epsilon_{a n n}$ is the luminosity produced by WIMPs annihilations per unit of baryonic mass. The factor $1 / 2(1 / 4)$ in the equation above is appropriate for self (not self) conjugate particles and $\langle\sigma v\rangle$ the velocity-averaged annihilation crosssection. If an equilibrium between capture and annihilation is reached the annihilation rates reduces to $\mathrm{A}=\mathrm{C} / 2$ and it is independent on the annihilation cross-section. The equilibrium timescale for such a processes, neglecting evaporation, is given by

$$
\tau_{\chi}=\left(\frac{1}{C A}\right)^{1 / 2} \sim\left(\frac{\pi^{3 / 2} r_{\chi}^{3}}{C\langle\sigma v\rangle}\right)^{1 / 2}
$$

and for annihilations cross-sections of the order $\langle\sigma v\rangle \sim$ $10^{-26} \mathrm{~cm}^{3} \mathrm{~s}^{-1}$, a typical value suggested by the requirement of the correct WIMP relic density, it is much shorter than the age of the Sun. In our calculations we compute the capture and annihilation terms self-consistently, and find good agreement with the equilibrium approximation for this value of the annihilations cross-section. However, we also explore scenarios with negligible or null annihilation cross-sections, as for the case of asymmetric-WIMPs, for which the equilibrium between annihilations and capture is not reached. 


\section{Evaporation}

WIMPs trapped inside the Sun may scatter off nuclei to velocities high enough to escape the gravitational field of the star and thus leave the system. Detailed studies on this phenomena $[80,81]$, called evaporation, have shown that the evaporation timescale, defined as the inverse of the evaporation rate, $E$ in Eq.1, depends exponentially on the mass of the WIMPs. Therefore, below a certain mass threshold, $m_{e v}$, basically all the WIMPs evaporate during the Sun evolution and conversely, slightly above $m_{e v}$ the evaporation is negligible. At high WIMP scattering cross sections, corresponding to the LTE regime, the evaporation starts to be inefficient since the WIMPs leaving the center of the star are rapidly re-scattered to low velocity orbits. The evaporation mass, depends therefore of the size and nature (SI or SD) of the scattering cross section. Considering the Sun, for $\sigma_{S D} \sim 10^{-36} \mathrm{~cm}^{2}$ it is typically of the order $m_{e v} \sim 4 \mathrm{GeV}$ and it decreases to $m_{e v} \sim 2 \mathrm{GeV}$ at $\sigma_{S D} \sim 10^{-34} \mathrm{~cm}^{2}$. In the following we focus to WIMP masses $m_{\chi} \geq 5 \mathrm{GeV}$ so the effect of the evaporation can be completely neglected for our purposes.

\section{Transport}

The energy (per unit mass, per unit time) transported by WIMPs can be cast as:

$$
\epsilon_{\mathrm{trans}}=\frac{1}{4 \pi r^{2} \rho(r)} \frac{\mathrm{d}}{\mathrm{d} r}\left[\mathfrak{f}(K) \mathfrak{h}(r) L_{\text {trans,LTE }}(r)\right] ;
$$

with

$$
\begin{aligned}
L_{\text {trans,LTE }}(r)= & 4 \pi r^{2} \kappa(r) n_{\chi, \mathrm{LTE}}(r) l(r) \\
& \times\left[\frac{\mathrm{k} T_{\star}(r)}{m_{\chi}}\right]^{1 / 2} \mathrm{k} \frac{\mathrm{d} T_{\star}(r)}{\mathrm{d} r}
\end{aligned}
$$

being the "transport luminosity" to be imputed to the WIMPs inside the Sun. The Knudsen number, $K$, indicates the "localization" of the WIMPs transport:

$$
K=\frac{l(0)}{r_{\chi}}
$$

The WIMP distribution $n_{\chi, \text { LTE }}$ is obtained in the approximation that the Knudsen number is very small and WIMPs are in local equilibrium with the baryons:

$$
n_{\chi, \mathrm{LTE}}(r)=n_{\chi, \mathrm{LTE}}(0)\left[\frac{T_{\star}(r)}{T_{\mathrm{c}}}\right]^{3 / 2}
$$

$$
\times \exp \left[-\int_{0}^{r} \frac{\mathrm{k} \alpha\left(r^{\prime}\right) \frac{\mathrm{d} T_{\star}\left(r^{\prime}\right)}{\mathrm{d} r^{\prime}}+m_{\chi} \frac{\mathrm{d} \phi\left(r^{\prime}\right)}{\mathrm{d} r^{\prime}}}{\mathrm{k} T_{\star}\left(r^{\prime}\right)} \mathrm{d} r^{\prime}\right]
$$

with the normalization $n_{\chi, \mathrm{LTE}}(0, t)$ obtained imposing $\int_{0}^{R_{\star}} 4 \pi r^{2} n_{\chi, \mathrm{LTE}}(r, t) \mathrm{d} r=N(t) . \quad T_{*}(r)$ is the temperature profile of the star and $\phi(r)$ is the local gravitational potential. The thermal diffusivity and conductivity coefficients, respectively $\kappa(r)$ and $\alpha(r)$, are defined as:

$$
\alpha(r)=\sum_{i} \frac{\sigma_{i} n_{i}(r)}{\sum_{j} \sigma_{j} n_{j}(r, t)} \alpha_{i}\left(\mu_{i}\right)
$$

and

$$
\kappa(r)=\left\{l(r) \sum_{i}\left[\kappa_{i}\left(\mu_{i}\right) l_{i}(r)\right]^{-1}\right\}^{-1} .
$$

with $\alpha_{i}\left(\mu_{i}\right)$ and $\kappa_{i}\left(\mu_{i}\right)$ obtained from tabulated values in Ref. [82]. The sum runs over all the nuclear species, $\mu_{i}$ is the WIMP-to-nucleus mass ratio and $n_{i}(r)$ is the number density of the species $i$ at any given radius.

The two correction factors which appear in Eq. A8 read:

$$
\mathfrak{h}(r) \approx\left(\frac{r-r_{\chi}}{r_{\chi}}\right)^{3}+1
$$

and

$$
\mathfrak{f}(K) \approx 1-\frac{1}{1+\mathrm{e}^{-\left(\ln K-\ln K_{0}\right) / \tau}}=1-\frac{1}{1+\left(\frac{K_{0}}{K}\right)^{1 / \tau}}
$$

with $\tau=0.5$ and $K_{0}=0.4$.

These two quantities, introduced by Gould \& Raffelt [82], extend the treatment of the WIMPs energy transport obtained under the assumption of local thermal equilibrium (i.e. small $K$ ), to the case of large Knudsen numbers. Therefore, the equations here described are virtually correct for any value of WIMP scattering cross-sections, applying both to local and non-local energy transport regimes. The WIMPs energy distribution in Eq. A11 differs from the analogue expression Eq. A4 valid for large $K$. In the following, for the computation of the annihilation rate, we adopt a distribution interpolating between the two regimes that we defined, following [7], as:

$$
n_{\chi}(r)=f(K) n_{\chi, \mathrm{LTE}}(r)+(1-f(K)) n_{\chi, n l} .
$$

As commented in Sec.A 2 this modification is however negligible in practice.
[1] Particle Dark Matter: Observations, Models and Searches, ed. G. Bertone, 2010, Cambridge University
Press; L. Bergstrom, Rept. Prog. Phys. 63, 793 (2000); G. Bertone, D. Hooper and J. Silk, Phys. Rep. 405 (2005) 
279

[2] P. Salati and J. Silk, ApJ 296, 679 (1985); A. Renzini, Astr. Ap. 171, 121 (1987); A. Bouquet and P. Salati, ApJ 346, 284 (1989);D. Dearborn, G. Raffelt, P. Salati, J. Silk and A. Bouquet, ApJ 354, 568 (1990);P. Salati, G. Raffelt and D. Dearborn, ApJ 357, 566 (1990)

[3] D. Dearborn, K. Griest and G. Raffelt, Astrophys. J. 368 (1991) 626.

[4] I.V. Moskalenko and L.L. Wai, Astrophys. J. 659:L29L32, 2007 [arXiv:astro-ph/0702654].

[5] G. Bertone and M. Fairbairn, Phys. Rev. D 77 (2008) 043515 [arXiv:0709.1485 [astro-ph]].

[6] M. Fairbairn, P. Scott and J. Edsjo, Phys. Rev. D 77 (2008) 047301

[7] P. Scott, M. Fairbairn and J. Edsjo, Mon. Not. Roy. Astron. Soc. 394, 82 (2008) [arXiv:0809.1871 [astro-ph]].

[8] Y. Ascasibar, A\&A, $462 \quad$ (2007) L65 arXiv:astro-ph/0612130 .

[9] D. Spolyar, K. Freese and P. Gondolo, Phys. Rev. Lett. 100 (2008) 051101.

[10] F. Iocco, Astrophys. J. 677 (2008) L1 [arXiv:0802.0941 [astro-ph]].

[11] K. Freese, D. Spolyar and A. Aguirre, 2008 [arXiv:0802.1724].

[12] K. Freese, P. Bodenheimer, D. Spolyar and P. Gondolo, 2008 [arXiv:0806.0617].

[13] F. Iocco, A. Bressan, E. Ripamonti, R. Schneider, A. Ferrara and P. Marigo, Mon. Not. Roy. Astron. Soc. 390 (2008) 1655 [arXiv:0805.4016 [astro-ph]].

[14] K. Freese, P. Gondolo, J.A. Sellwood and D. Spolyar, [arXiv:0805.3540].

[15] M. Taoso, G. Bertone, G. Meynet and S. Ekstrom, Phys. Rev. D 78 (2008) 123510 [arXiv:0806.2681 [astro-ph]].

[16] S. C. Yoon, F. Iocco and S. Akiyama, Astrophys. J. 688 (2008) L1 [arXiv:0806.2662 [astro-ph]].

[17] E. Ripamonti, F. Iocco, A. Bressan, R. Schneider, A. Ferrara and P. Marigo, PoS IDM2008 (2009) 075 [arXiv:0903.0346 [astro-ph.CO]]; E. Ripamonti, F. Iocco, A. Ferrara, R. Schneider, A. Bressan and P. Marigo, MNRAS in press, arXiv:1003.0676 [astro-ph.CO].

[18] I. Lopes and J. Silk, Phys. Rev. Lett. 88, 151303 (2002) [arXiv:astro-ph/0112390].

[19] I. P. Lopes, G. Bertone and J. Silk, Mon. Not. Roy. Astron. Soc. 337, 1179 (2002) [arXiv:astro-ph/0205066].

[20] A. Bottino, G. Fiorentini, N. Fornengo, B. Ricci, S. Scopel and F. L. Villante, Phys. Rev. D 66, 053005 (2002) [arXiv:hep-ph/0206211].

[21] R.Bernabei et al., Riv.Nuovo Cim.26N1 (2003) 1.

[22] C. E. Aalseth et al. [CoGeNT collaboration], arXiv:1002.4703 [astro-ph.CO].

[23] Z. Ahmed et al. [The CDMS-II Collaboration], arXiv:0912.3592 [astro-ph.CO].

[24] D. N. Spergel and P. J. Steinhardt, Phys. Rev. Lett. 84 (2000) 3760 [arXiv:astro-ph/9909386].

[25] M. T. Frandsen and S. Sarkar, arXiv:1003.4505 [hep-ph].

[26] D. Tucker-Smith and N. Weiner, Phys. Rev. D 64 (2001) 043502 [arXiv:hep-ph/0101138].

[27] A. Gould, ApJ 567 (1987) 532.

[28] R. Catena and P. Ullio, arXiv:0907.0018 [astro-ph.CO].

[29] P. Salucci, F. Nesti, G. Gentile and C. F. Martins, arXiv:1003.3101 [astro-ph.GA].

[30] M. Weber and W. de Boer, arXiv:0910.4272 [astroph.CO].

[31] M. Pato, O. Agertz, G. Bertone, B. Moore and
R. Teyssier, arXiv:1006.1322 [astro-ph.HE].

[32] T. Bruch, A. H. G. Peter, J. Read, L. Baudis and G. Lake, Phys. Lett. B 674 (2009) 250 [arXiv:0902.4001 [astroph.HE]].

[33] E. Aprile et al. [XENON100 Collaboration and XENON100 Collaboration and XENON100 Collabo], arXiv:1005.0380 [astro-ph.CO].

[34] S. Archambault et al., Phys. Lett. B 682 (2009) 185 [arXiv:0907.0307 [hep-ex]].

[35] R.Bernabei et al., Eur.Phys. J.C56 (2008) 333.

[36] R. Bernabei et al., J. Phys. Conf. Ser. 203 (2010) 012003.

[37] C. Savage, K. Freese, P. Gondolo and D. Spolyar, JCAP 0909 (2009) 036 [arXiv:0901.2713 [astro-ph]].

[38] J. Kopp, T. Schwetz and J. Zupan, JCAP 1002 (2010) 014 [arXiv:0912.4264 [hep-ph]].

[39] A. Bottino, F. Donato, N. Fornengo and S. Scopel, arXiv:0912.4025 [hep-ph].

[40] D. Hooper, J. March-Russell and S. M. West, Phys. Lett. B 605 (2005) 228 [arXiv:hep-ph/0410114].

[41] D. E. Kaplan, M. A. Luty and K. M. Zurek, Phys. Rev. D 79 (2009) 115016 [arXiv:0901.4117 [hep-ph]].

[42] F. Sannino and R. Zwicky, Phys. Rev. D 79, 015016 (2009) [arXiv:0810.2686 [hep-ph]].

[43] G. D. Kribs, T. S. Roy, J. Terning and K. M. Zurek, arXiv:0909.2034 [hep-ph].

[44] J. L. Feng, M. Kaplinghat, H. Tu and H. B. Yu, JCAP 0907 (2009) 004 [arXiv:0905.3039 [hep-ph]].

[45] A. R. Zentner, Phys. Rev. D 80 (2009) 063501 [arXiv:0907.3448 [astro-ph.HE]].

[46] A. Kusenko and M. E. Shaposhnikov, Phys. Lett. B 418 (1998) 46 [arXiv:hep-ph/9709492].

[47] G. R. Farrar, Phys. Rev. Lett. 53 (1984) 1029.

[48] B. D. Wandelt, R. Dave, G. R. Farrar, P. C. McGuire, D. N. Spergel and P. J. Steinhardt, arXiv:astro-ph/0006344.

[49] R. N. Mohapatra, S. Nussinov and V. L. Teplitz, Phys. Rev. D 66 (2002) 063002 [arXiv:hep-ph/0111381].

[50] Z. Berezhiani, D. Comelli and F. L. Villante, Phys. Lett. B 503 (2001) 362 [arXiv:hep-ph/0008105].

[51] R. Foot and R. R. Volkas, Phys. Rev. D 70 (2004) 123508 [arXiv:astro-ph/0407522].

[52] R. Foot, Phys. Rev. D 81 (2010) 087302 [arXiv:1001.0096 [hep-ph]].

[53] S. W. Randall, M. Markevitch, D. Clowe, A. H. Gonzalez and M. Bradac, arXiv:0704.0261 [astro-ph].

[54] M. Taoso, G. Bertone and A. Masiero, JCAP 0803 (2008) 022 [arXiv:0711.4996 [astro-ph]].

[55] P. Eggenberger, G. Meynet, A. Maeder, R Hirschi, C. Charbonnel, S. Talon, S. Ekstrom, Astrophysics and Space Science 316 (2008) 43.

[56] M. Asplund, N. Grevesse, A.J. Sauval, Cosmic Abundances as Records of Stellar Evolution and Nucleosynthesis in honor of David L. Lambert, ASP Conference Series 336 (2005) 25.

[57] J. N. Bahcall and M. H. Pinsonneault, Reviews of Modern Physics 64 (1992) 885.

[58] J. N. Bahcall Phys. Rev. C65, 015802 (2002)

[59] B. Aharmim et al. [SNO Collaboration], arXiv:0910.2984 [nucl-ex].

[60] C. Arpesella et al. [The Borexino Collaboration], Phys. Rev. Lett. 101 (2008) 091302 [arXiv:0805.3843 [astro$\mathrm{ph}]$.

[61] A. Serenelli, S. Basu, J. W. Ferguson and M. Asplund, arXiv:0909.2668 [astro-ph.SR]. 
[62] C. Pena-Garay and A. Serenelli, arXiv:0811.2424 [astro$\mathrm{ph}$.

[63] J. N. Bahcall and A. M. Serenelli, Astrophys. J. 626, 530 (2005) [arXiv:astro-ph/0412096].

[64] M. Asplund, N. Grevesse, A. J. Sauval and P. Scott, Ann. Rev. Astron. Astrophys. 47 (2009) 481 [arXiv:0909.0948 [astro-ph.SR]].

[65] J. Casanellas and I. Lopes, Astrophys. J. 705 (2009) 135 [arXiv:0909.1971 [astro-ph.CO]].

[66] A. Bolanos, O. G. Miranda, A. Palazzo, M. A. Tortola and J. W. F. Valle, Phys. Rev. D 79 (2009) 113012 [arXiv:0812.4417 [hep-ph]].

[67] J. N. Bahcall, A. M. Serenelli and S. Basu, Astrophys. J. Suppl. 165 (2006) 400 [arXiv:astro-ph/0511337].

[68] F. L. Villante and B. Ricci, Astrophys. J. 714, 944 (2010) [arXiv:0912.4696 [astro-ph.SR]].

[69] D. T. Cumberbatch, J. A. Guzik, J. Silk, L. S. Watson and S. M. West, arXiv:1005.5102 [astro-ph.SR].

[70] Z. Thomas, D. Tucker-Smith and N. Weiner, Phys. Rev. D 77 (2008) 115015 [arXiv:0712.4146 [hep-ph]].

[71] N. Arkani-Hamed, D. P. Finkbeiner, T. R. Slatyer and N. Weiner, Phys. Rev. D 79, 015014 (2009)
[arXiv:0810.0713 [hep-ph]].

[72] Y. Cui, D. E. Morrissey, D. Poland and L. Randall, JHEP 0905, 076 (2009) [arXiv:0901.0557 [hep-ph]].

[73] S. Chang, G. D. Kribs, D. Tucker-Smith and N. Weiner, Phys. Rev. D 79 (2009) 043513 [arXiv:0807.2250 [hep$\mathrm{ph}]$.

[74] J. March-Russell, C. McCabe and M. McCullough, JHEP 0905, 071 (2009) [arXiv:0812.1931 [astro-ph]].

[75] C. Arina, F. S. Ling and M. H. G. Tytgat, JCAP 0910, 018 (2009) [arXiv:0907.0430 [hep-ph]].

[76] K. Schmidt-Hoberg and M. W. Winkler, JCAP 0909, 010 (2009) [arXiv:0907.3940 [astro-ph.CO]].

[77] S. Nussinov, L. T. Wang and I. Yavin, JCAP 0908 (2009) 037 [arXiv:0905.1333 [hep-ph]].

[78] A. Menon, R. Morris, A. Pierce and N. Weiner, arXiv:0905.1847 [hep-ph].

[79] K.Griest and D.Seckel, Nucl. Phys. B 296 (1987) 681.

[80] A. Gould, Astrophys. J. 321 (1987) 560.

[81] A.Gould, Astrophys. J. 356 (1990) 302.

[82] A. Gould and G. Raffelt, Astrophys. J. 352 (1990) 654. 